gr-qc/9309024, CGPG-93/9-3

\title{
Reality Conditions in Nonperturbative Quantum Cosmology
}

\author{
Guillermo A. Mena Marugán \\ Center for Gravitational Physics and Geometry, \\ The Pennsylvania State University, 104 Davey Laboratory, \\ University Park, PA 16802-6300, USA. \\ On leave from: Instituto de Matemáticas y Física Fundamental, \\ C.S.I.C., Serrano 121, 28006 Madrid, Spain. \\ September, 1993
}

\begin{abstract}
Abstrat
We carry out the nonperturbative canonical quantization of several types of cosmological models that have already been studied in the geometrodynamic formulation using the complex path-integral approach. We establish a relation between the choices of complex contours in the path integral and the sets of reality conditions for which the metric representation is well defined, proving that the ambiguity in the selection of complex contours disappears when one imposes suitable reality conditions. In most of the cases, the wave functions defined by means of the path integral turn out to be non-normalizable and cannot be accepted as proper quantum states. Moreover, the wave functions of the Universe picked out in quantum cosmology by the no-boundary condition and the tunneling proposals do not belong, in general, to the Hilbert space of quantum states. Finally, we show that different sets of reality conditions can lead to equivalent quantum theories. This fact enables us to extract physical predictions corresponding to Lorentzian gravity from quantum theories constructed with other than Lorentzian reality conditions.
\end{abstract}




\section{Introduction}

The nonperturbative canonical quantization programme proposed by Ashtekar $[1,2]$ is accepted nowadays as one of the most promising approaches to construct a consistent theory of quantum gravity. In spite of the success of this programme, developed systematically over the last seven years $[2,3]$, the nonperturbative quantization of the full theory of general relativity remains still incomplete. In order to gain insight into the kind of difficulties involved in quantizing gravitational systems, an increasing number of works in the literature have been devoted to the nonperturbative quantization 7 of truncated models in gravity [4,5]. Apart from providing a good arena to test the applicability of the quantization procedure, the completion of this nonperturbative quantization in minisuperspaces of cosmological interest is clearly relevant inasmuch as it allows us to extract physical predictions in quantum cosmology [5].

A particularly appealing possibility consists in attempting the nonperturbative quantization of minisuperspace models that have been previously studied in quantum cosmology using the standard techniques of the geometrodynamic formulation [6]. In this way, one could check the validity of the results obtained in quantum cosmology and reach a better physical interpretation of the mathematical framework employed in the nonperturbative quantization, eventually adopting proposals from the geometrodynamic formulation to solve current problems in nonperturbative quantum gravity.

With these motivations in mind, we will carry out in this work the nonperturbative quantization of two families of minisuperspace models that have been considered in the literature as examples of exactly soluble systems in the complex path-integral formalism of quantum cosmology.

\footnotetext{
*In the following, we will understand "nonperturbative quantization" to refer to the result of the nonperturbative canonical quantization programme proposed by Ashtekar [2].
} 
First, we will study a class of homogeneous and spherically symmetric models provided with a minimally coupled massless scalar field with exponential potential. Models of this kind appear, for instance, in the dimensional reduction of fivedimensional Kaluza-Klein gravity with cosmological constant $[7,8]$, and their classical solutions display, in general, either exponential or power-law inflation $[8,9,10]$. The path-integral analysis of these models was discussed in detail in Ref. [10].

The second type of models that we will analyze are a family of homogeneous and anisotropic minisuperspaces with cosmological constant, that contains the locally rotationally symmetric (LRS) Bianchi types I and III and the Kantowski-Sachs model as particular cases. The complex path integral between fixed metric configurations was thoroughly studied for these minisuperspaces in Ref. [11].

For all the above models, we will implement to completion the nonperturbative canonical quantization programme [2]. We briefly recall that, in order to apply this quantization procedure to a system with constraints, one first selects a complete set of complex functions on phase space that is closed under Poisson brackets $[2,3]$. This set is promoted to a $\star$-algebra of elementary operators, capturing the complex conjugation relations between classical variables as $\star$-relations $[2,12]$. The $\star$-algebra of elementary operators is then represented on a chosen vector space, with the quantum states provided by the kernel of all the operators that represent the classical constraints of the system. Finally, one can fix the inner product in the space of quantum states by imposing the $\star$-relations between elementary operators as adjointness conditions [13]. These adjointness requirements are usually called reality conditions $[2]$.

Reality conditions seem to play a double role in the nonperturbative canonical quantization programme: they simultaneously select the quantum theory associated with a specific section of the complex phase space of the system (the section chosen by reality conditions when considered as complex conjugation relations $[2,12]$ ) 
and determine the inner product in that theory by demanding some adjointness requirements.

There is a certain parallel between the need to consider complex functions on phase space in the nonperturbative quantization, and the definition of the Euclidean path integral in quantum cosmology as an integral over complex fields and metrics. In the nonperturbative quantization, reality conditions remove the ambiguity that was introduced by the complexification of the phase space. In path-integral cosmology $[14,15]$, on the other hand, the complex contours of integration are severely restricted by the requirement of convergence when one imposes the reality of the metric and matter fields on the boundaries of the manifold where one is integrating [16]. Nevertheless, the ambiguity in this latter case is only partially removed, and more than one choice of inequivalent complex contours of integration are usually acceptable (even if one tries to demand additional conditions for the path integral [16]).

Using that, for the models considered in this work, both the nonperturbative quantization and the path integral between fixed geometrodynamic configurations can be carried out successfully, we will show that the possible choices of complex contours in the geometrodynamic path integral correspond in fact to the selection of different reality conditions for the system. In particular, once one has fixed an adequate set of reality conditions, the complex contours of integration turn out to be essentially unique.

We will also explore another related topic that at present remains unclear in the nonperturbative programme: the relation between the quantum theories obtained by demanding different sets of reality conditions. In general, one of the main technical difficulties encountered in the implementation of the nonperturbative quantization scheme is the imposition of the reality conditions associated with Lorentzian gravity (specially in Ashtekar variables [12]). A tentative way out of this problem could 
consist of completing the nonperturbative quantization by employing simpler reality conditions, and recovering somehow the Lorentzian predictions from the quantum theory so constructed. For the models studied in this paper, we will prove that sets of non-Lorentzian reality conditions can lead in some cases to quantum theories that are equivalent to that corresponding to Lorentzian gravity, so that one can actually extract from them all the relevant physics.

\section{Minisuperspaces: Nonperturbative Canonical Quantization}

\subsection{Isotropic models with scalar field}

The first class of minisuperspaces that we want to consider are a family of homogeneous and isotropic models with a minimally coupled scalar field. We will restrict the metric of these models to be of the form

$$
d s^{2}=-N^{2} \frac{d t^{2}}{a^{2}(t)}+a^{2}(t) d \Omega_{3}^{2},
$$

and the scalar field to be homogeneous and with a potential of exponential type:

$$
V(\phi)=\alpha \cosh (2 \phi)+\beta \sinh (2 \phi)
$$

Here, $d \Omega_{3}^{2}$ is the metric on the unit three-sphere, $\alpha$ and $\beta$ are real constants, and $N$, $a$ and $\phi$ denote, respectively, the numerical values of the lapse function, the scale factor and the scalar field (in the system of units chosen in Ref. [10]).

In the new variables

$$
x=a^{2} \cosh (2 \phi), \quad y=a^{2} \sinh (2 \phi),
$$

the Hamiltonian constraint of these minisuperspaces takes the simple expression

$$
\mathcal{H}=\frac{1}{2}\left(-4 p_{x}^{2}+4 p_{y}^{2}+\alpha x+\beta y-1\right)=0,
$$

where $p_{x}$ and $p_{y}$ are the momenta canonically conjugate to $x$ and $y$. 
We notice that the transformation (3) maps the "physical" region $a, \phi \in \mathbb{R}$ into the light cone of the origin in the real $x y$ space. However, instead of restricting the domain of the variables $x$ and $y$, we will regard them as our elementary complex configuration variables, following, in that sense, the same quantization approach that was adopted in Ref. [10].

On the other hand, all models with $\alpha \neq \beta$ can be related to the case $\beta=0$ by a linear transformation of coordinates [10]. It will then suffice to concentrate our attention on two types of scalar field potential: those corresponding to $\beta=0$ and to $\alpha=\beta$. We will require in addition that $\alpha>0$, so that the potential for the scalar field is at least bounded from below. Finally, we will employ from now on the notation

$$
k=\frac{1}{\alpha}
$$

so, $k>0$ for all the models under consideration.

\subsubsection{The cosh potential}

Let us analize first the case $\beta=0, \alpha=k^{-1}>0$. For this model, it is easy to check that the variables

$$
Q=y+8 k p_{x} p_{y}, \quad P=p_{y}
$$

form a pair of canonically conjugate observables, i.e., of functions on phase space that commute with the Hamiltonian constraint. Introducing then the additional coordinates

$$
H=x+4 k\left(p_{y}^{2}-p_{x}^{2}\right), \quad T=p_{x}
$$

we obtain a complete set of (complex) phase-space variables. Equations (6) define a canonical transformation of coordinates that is analytic and invertible everywhere. This transformation is generated by the function

$$
F=x T+y P+4 k P^{2} T-\frac{4}{3} k T^{3} .
$$


The variable $H$ is essentially equal to the Hamiltonian of the system

$$
\mathcal{H} \propto H-k=0
$$

The coordinate $T$, on the other hand, plays the role of an intrinsic time, since $T$ and $H$ are canonically conjugate to each other.

Given the simplicity of the Hamiltonian constraint and the Poisson brackets in the new coordinates (6), it is now almost straightforward to achieve the nonperturbative quantization of this minisuperspace. We choose $Q, P, H$ and $T$ as our set of basic functions on phase space, and represent the corresponding algebra of elementary operators on the space of distributions in the complex variables $T$ and $P$ (this choice of representation will be very convenient for our later discussion in section 3 ).

In the adopted representation (and taking $\hbar=1$ from now on), the action of the elementary operators can be defined in the following way:

$$
\hat{P} \Phi=P \Phi(T, P), \quad \hat{Q} \Phi=i \frac{\partial \Phi}{\partial P}(T, P), \quad \hat{T} \Phi=T \Phi(T, P), \quad \hat{H} \Phi=i \frac{\partial \Phi}{\partial T}(T, P),
$$

and the solutions to the Hamiltonian constraint are simply given by the expression

$$
\Phi(T, P)=e^{-i k T} f(P)
$$

In order to complete the nonperturbative quantization, we have to impose a set of reality conditions, and find the associated inner product. For Lorentzian gravity, the variables $Q, P, H$ and $T$ are real, and thus we conclude that

$$
\hat{Q}^{\star}=\hat{Q}, \quad \hat{P}^{\star}=\hat{P}, \quad \hat{H}^{\star}=\hat{H}, \quad \hat{T}^{\star}=\hat{T} .
$$

Among these relations, only the two first can be promoted to self-adjointness conditions in the quantum theory, because $\hat{H}$ and $\hat{T}$ are not observables and, therefore, their action in the space of quantum states is not well defined. Nevertheless, requiring that $\hat{Q}$ and $\hat{P}$ be self-adjoint will be enough to fix the inner product, since 
these variables form a complete set of observables for the model [3,13]. On the other hand, if we insist on the connection between complex conjugation and $\star$-relations, the conditions on $\hat{H}$ and $\hat{T}$ in (11) allow us to restrict the domain of the variable $T$ to be the real axis. This restriction is consistent with the Lorentzian dynamics, for $\dot{T}=N \alpha\{H, T\}=N \alpha$ is always real if $N \in \mathbb{R}$.

To determine the inner product, we first notice that the dependence of the quantum states on $T$ is entirely fixed (each state is completely characterized by the distribution $f(P)$ ). As a consequence, the inner product can be made time independent by choosing an adequate $T$-dependence in the integration measure. In this way, one arrives at a product of the form

$$
<\Psi \mid \Phi>=\frac{i}{2} \int d P \wedge d \bar{P} \mu(P, \bar{P}) \overline{h(P)} f(P)
$$

where $\Psi=\exp (-i k T) h(P)$, the symbol ${ }^{-}$denotes complex conjugation and the measure $\mu(P, \bar{P})$ must be such that $\hat{Q}$ and $\hat{P}$ are self-adjoint. After a short calculation, we conclude that

$$
<\Psi \mid \Phi>=\int_{\mathbb{R}} d P \bar{h}(P) f(P),
$$

so that the Hilbert space of quantum states is $L^{2}(\mathbb{R})$.

Before continuing our discussion, we would like to make a few remarks on the quantum theory that we have constructed. We have seen above that, for Lorentzian gravity, the variables $x$ and $y$ in (3) should be not only real, but restricted to the light cone of the origin. However, we have carried out the quantization without imposing the analogue of this constraint in the new variables (6). In this sense, the quantum theory that we have obtained contains contributions from metrics and scalar fields that are not purely Lorentzian. One reason that justifies this approach is simply that the classical evolution associated to the Hamiltonian (4) does not leave invariant the light cone of the origin in the real $x y$ plane; thus, if we take $x$ and $y$ as the basic configuration variables of the model, we cannot consistently 
restrict their domain to the proposed region. On the other hand, our main interest lies in the comparison between the results of the nonperturbative quantization and the complex path-integral formalism. Since the path-integral analysis of this model was performed without restricting the domain of the variables $x$ and $y$ [10], we will adopt here the same kind of strategy in order to maintain the parallel in the quantization as far as possible.

Let us study now more general sets of reality conditions than those corresponding to Lorentzian gravity. For our purposes in section 3, where we will explore the relation between complex contours for the path integral and reality conditions, it will suffice to consider conditions of the type

$$
\begin{aligned}
& \hat{T}^{\star}=e^{-i 2 \varphi} \hat{T}, \quad \hat{H}^{\star}=e^{i 2 \varphi} \hat{H}+k\left(1-e^{i 2 \varphi}\right), \\
& \hat{Q}^{\star}=e^{-i 2 \theta} \hat{Q}, \quad \hat{P}^{\star}=e^{i 2 \theta} \hat{P}-i \epsilon\left(1+e^{i 2 \theta}\right),
\end{aligned}
$$

where $\epsilon \in \mathbb{R}$ and $\varphi, \theta \in[0, \pi)$ are three real constants. Note that the $\star$-relations $(14,15)$ are compatible with the commutation relations, provided that

$$
(a \hat{X}+b \hat{Y})^{\star}=\bar{a} \hat{X}^{\star}+\bar{b} \hat{Y}^{\star}, \quad(\hat{X} \hat{Y})^{\star}=\hat{Y}^{\star} \hat{X}^{\star}
$$

for any complex constants $a$ and $b$ and any operators $\hat{X}$ and $\hat{Y}$ in the $\star$-algebra. The $\star$-relation for $\hat{H}$ has been chosen in such a way that the operator version of the Hamiltonian constraint $(8)$ and its $\star$-analogue are equivalent. In the $(T, P)$ representation, conditions (14) can be interpreted as restricting the domain of the variable $T$ to lie in the real axis rotated by an angle $\varphi$ :

$$
T \in \Gamma \equiv\left\{e^{i \varphi} \tau, \quad \tau \in \mathbb{R}\right\}, \quad \varphi \in[0, \pi) .
$$

This is clearly compatible with the Hamiltonian dynamics for non-Lorentzian times obtained by a similar rotation of the lapse function $(N=\exp (i \varphi) c$ with $c \in \mathbb{R})$, since $\dot{T}=N \alpha$. 
Following similar arguments to those presented above for the Lorentzian reality conditions, it is not difficult to conclude that the inner product determined by conditions (15), imposed as self-adjointness relations in the operators $\hat{Q}$ and $\hat{P}$, takes the simple expression

$$
<\Psi \mid \Phi>=e^{i \theta} \int_{\gamma} d P \overline{h(P)} f(P)
$$

where $\gamma$ is an infinite line in the complex $P$ plane that can be parametrized as

$$
\gamma \equiv\left\{e^{-i \theta} p+i \epsilon, \quad p \in \mathbb{R}\right\}
$$

and $\theta$ and $\epsilon$ are the same constants that appear in equation (15). From now on, we will denote the corresponding Hilbert space as $L^{2}(\gamma)$.

\subsubsection{The exponential potential}

We turn now to the case of a purely exponential potential for the scalar field: $\alpha=\beta>0$. For this model, the following transformation of phase-space coordinates provides a new set of canonical variables:

$$
\begin{array}{cl}
Q=y+4 k p_{y}^{2}-k\left(p_{y}-p_{x}\right)^{2}, & P=p_{y}-p_{x} \\
H=x+y+4 k\left(p_{y}^{2}-p_{x}^{2}\right), & T=p_{x} .
\end{array}
$$

This canonical transformation is generated by the function

$$
F=\frac{P}{2}(y-x-H)-\frac{1}{16 k P}(x+y-H)^{2} .
$$

We note that the transformation (20) is analytic and invertible everywhere, even though the generator $(21)$ is singular at $P=0$ (this singularity will nevertheless have some consequences in the $x y$ representation, as we will see in the following section). 
Just as in the $\beta=0$ model, the variables $Q$ and $P$ are a canonically conjugate pair of observables, $H$ is essentially the Hamiltonian, which is given again by equation (8), and $T$ corresponds to an intrinsic time. To quantize the model, we choose the space of distributions in the variables $P$ and $H$ as our representation space, and introduce the following action for the elementary operators

$$
\hat{P} \Phi=P \Phi(H, P), \quad \hat{Q} \Phi=i \frac{\partial \Phi}{\partial Q}(H, P), \quad \hat{H} \Phi=H \Phi(H, P), \hat{T} \Phi=-i \frac{\partial \Phi}{\partial H}(H, P),
$$

with the derivatives defined in the distributional sense. In this representation, the general solution to the Hamiltonian constraint adopts the compact expression

$$
\Phi(H, p)=\delta(H-k) f(P),
$$

where $\delta$ is the Dirac function and $f(P)$ any distribution.

To fix the inner product, we still have to impose an admissible set of reality conditions. We will first concentrate our attention on reality conditions of the type $(14,15)$. The inner product can be clearly made $H$ independent, because all quantum states (23) are characterized by their dependence on $P$. The set of reality conditions (15), imposed as adjointness relations among the observables of the theory, determine then a unique inner product of the form (18). In particular, the inner product for Lorentzian gravity (corresponding to conditions (11)) is given again by equation (13).

Another possibility that we want to investigate is that of quantizing the theory assuming a restricted domain for the variable $P$. This quantization approach is consistent in principle, since the classical evolution leaves invariant any domain of definition for $P$. We are particularly interested (for our later discussion in section 3 ) in studying those cases in which $P$ is restricted to a half-infinite segment with endpoint at $P=0$ :

$$
P \in \gamma_{+} \equiv\left\{e^{-i \theta} p, p \in \mathbb{R}^{+}\right\}
$$


with $\theta \in[0,2 \pi)$ a fixed angle. For this kind of models, the reduced phase space can be taken to be the cotangent bundle over $\gamma_{+}$. An adequate set of elementary variables in that reduced phase space is provided by $P$ and the new coordinate

$$
q=P Q
$$

where $Q$ is the momentum canonically conjugate to $P$. The choice of $q$ as the generalized momentum variable is motivated by the fact that its associated vector field, $P(\partial / \partial P)$, is complete on $\gamma_{+}$, while the vector field that correspond to $Q$, $(\partial / \partial P)$, fails to satisfy this condition [5].

To quantize the system, we choose $(H, T, P, q)$ as our set of elementary variables, which is obviously closed under the Poisson-bracket structure. We then adopt the same $(H, P)$ representation that was selected before in this subsection, except that we substitute now the operator $\hat{Q}$ in (22) by the new elementary operator

$$
\hat{q} \Phi=i P \frac{\partial \Phi}{\partial P}(H, P) .
$$

The general solution to the Hamiltonian constraint is still given by equation (23). On the other hand, we impose as reality conditions that, in addition to relations $(14)$,

$$
\hat{q}^{\star}=\hat{q}, \quad \hat{P}^{\star}=e^{i 2 \theta} \hat{P} .
$$

The second of these conditions is the $\star$-analogue of the complex conjugation relation derived from (24). The first condition in (27) has been chosen to guarantee that the $\star$-relations (that must satisfy the requirements (16)) are compatible with the commutator $[\hat{q}, \hat{P}]=i \hat{P}$.

Generalizing our previous analysis of the implementation of the reality conditions, it is straightforward to arrive at an inner product of the form

$$
<\Psi \mid \Phi>=\int_{\gamma_{+}} \frac{d P}{P} \overline{h(P)} f(P),
$$


with $\gamma_{+}$the half-infinite contour defined in (24), and $\Psi=\delta(H-k) h(P)$. The Hilbert space of quantum states is thus $L^{2}\left(\gamma_{+}, P^{-1} d P\right)$.

We finally notice that the quantum theory that we have constructed is in fact unitarily equivalent to that with Hilbert space equal to $L^{2}\left(\gamma_{+}\right)$(the Hilbert space with inner product given by (18) evaluated at $\gamma=\gamma_{+}$) and operator $\hat{q}$ defined as

$$
\hat{q} \tilde{\Phi}=i\left(P \frac{\partial}{\partial P}+\frac{1}{2}\right) \tilde{\Phi}(H, P) .
$$

The isomorphism between the Hilbert spaces $L^{2}\left(\gamma_{+}, P^{-1} d P\right)$ and $L^{2}\left(\gamma_{+}\right)$is provided by

$$
\begin{gathered}
\Phi(H, P)=\delta(H-k) f(P) \stackrel{I}{\longrightarrow} \tilde{\Phi}(H, P)=\delta(H-k) \frac{f(P)}{\sqrt{P}} \\
f(P) \in L^{2}\left(\gamma_{+}, P^{-1} d P\right) ; \quad \tilde{f}(P)=\frac{f(P)}{\sqrt{P}} \in L^{2}\left(\gamma_{+}\right) .
\end{gathered}
$$

The action of the operator $\hat{q}$ in (29) corresponds, on the other hand, to the symmetrized product of the operators $\hat{Q}$ and $\hat{P}$, as given by equation (22). In this sense, the quantum theory associated with reality conditions (14) and (27) is equivalent to that with elementary operators $(\hat{H}, \hat{T}, \hat{P}, \hat{Q})$ defined through $(22)$ and Hilbert space $L^{2}\left(\gamma_{+}\right)$. We will use this alternative quantization in the next section for our discussion of the connection between choices of complex contours for the path integral and sets of reality conditions.

\subsection{Anisotropic models}

In addition to the previous models, we want to analyze also a class of anisotropic minisuperspaces with cosmological constant whose spacetime metric can be expressed in the generic form

$$
d s^{2}=-N^{2} \frac{d t^{2}}{a^{2}(t)}+a^{2}(t) d r^{2}+b^{2}(t) d \Omega_{2}^{2} .
$$

Here, $N, a$ and $b$ are the numerical values of the lapse function and the two scale factors of the model (in adequate units, see Ref. [11]), $d \Omega_{2}^{2}$ denotes the metric on 
a compact orientable two-manifold of constant scalar curvature equal to $2 k$, and $k=+1,0$ or -1 [11]. Depending on the value of $k$, the metric (31) describes either the Kantowski-Sachs model $(k=1)$, the LRS Bianchi type I $(k=0)$ or the LRS Bianchi type III $(k=-1)$.

After defining the new variable

$$
c=a^{2} b,
$$

the Hamiltonian constraint of these systems can be written

$$
\mathcal{H}=\frac{1}{2}\left(-4 p_{c} p_{b}+\lambda b^{2}-k\right)=0,
$$

where $\lambda$ is the rescaled cosmological constant [11], and $p_{c}$ and $p_{b}$ are the momenta canonically conjugate to $c$ and $b$.

Paralleling the analysis of the isotropic models with a scalar field, we introduce the following canonical transformation of variables

$$
\begin{array}{ll}
Q=c+\frac{\lambda b^{3}-6 b p_{b} p_{c}}{6 p_{c}^{2}}, & P=p_{c}, \\
H=-4 p_{c} p_{b}+\lambda b^{2}, & T=\frac{b}{4 p_{c}} .
\end{array}
$$

A generating function for the above transformation is given by

$$
F=c P+\frac{\lambda b^{3}-3 b H}{12 P} .
$$

Once again, the variables $Q$ and $P$ provide a conjugate pair of observables, the Hamiltonian constraint adopts the simple expression (8) and $T$ plays the role of an intrinsic time. The only relevant difference with respect to the minisuperspaces studied before is the existence of a singularity in the canonical transformation (34) at $P=p_{c}=0$. We will return to this point at the end of this section.

To achieve the nonperturbative canonical quantization of these models, we choose $(Q, P, H, T)$ as our set of elementary complex variables, take the space of distributions in $H$ and $P$ as our representation space, and define the action of the elementary 
operators as in equation (22). The general solution to the Hamiltonian constraint is then of the form (23), with the parameter $k$ equal to either 1,0 or -1 .

The same line of reasoning that was presented above for the scalar field model with exponential potential leads us to conclude that, for reality conditions of the type $(14,15)$, or for conditions (14) and (27) if the range of the variable $P$ is restricted to lie on a half-infinite contour, the inner product is unique and given by formula (18) or (28), respectively. Therefore, the Hilbert space of quantum states is again equal to either $L^{2}(\gamma)$ or $L^{2}\left(\gamma_{+}\right)$(assuming that we adopt for both classes of reality conditions the representation defined through equation (22)).

The only caveat with respect to this result is the existence of a singularity at the origin of $P$ in the canonical transformation (34). This singularity may affect the quantum theories with Hilbert spaces of the form $L^{2}(\gamma(\epsilon=0))$, where $\gamma(\epsilon=0)$ (as given by (19)) is a contour obtained by rotating the real axis around the origin, and therefore contains the point $P=0 \rrbracket$. Nevertheless, we notice that one can always avoid getting contributions from the singularity at the origin of $P$ by requiring that $f(P=0)=0$ for all quantum states $f$. This condition can be consistently imposed on any $f \in L^{2}(\gamma(\epsilon=0))$, because a function in the Hilbert space $L^{2}(\gamma)$ is defined only almost everywhere in $\gamma$.

\section{Complex Path Integral and Reality Conditions}

In the previous section, we have completed the nonperturbative quantization of the considered minisuperspace models by using a set of canonically conjugate variables that mix the geometrodynamic configuration and momenta coordinates. We now want to analyze the relation between the representation employed in this nonperturbative quantization and the metric representation $\$$, discussing thereafter the validity

\footnotetext{
${ }^{\dagger}$ Note that all the contours with $\theta=\pi / 2$ in (19) are equivalent to $\gamma(\epsilon=0, \theta=\pi / 2$ ).

${ }^{\ddagger}$ From now on, we will use the term "metric representation" to designate any representation in which the metric and the matter fields of the model act as multiplicative operators.
} 
of the complex path-integral approach in geometrodynamics. In particular, we will show that the selection of different complex contours in the path integral correspond in fact to the choice of different sets of reality conditions for the nonperturbative quantization.

\subsection{Scalar field with cosh potential}

Let us study first the case of the homogeneous and spherically symmetric model provided with a scalar field with hyperbolic cosine potential. It is not difficult to check that, for this minisuperspace, the change from the representation $(T, P)$, defined through equation (9), to the metric representation $(x, y)$ used in Ref. [10] is obtained by means of the transformation

$$
f(x, y)=\int_{\gamma} d P \int_{\Gamma} d T e^{i F(x, y, T, P)} \Phi(T, P)
$$

where $F$ is the generating function that appears in $(7), \Phi(T, P)$ are the quantum states (10), and $\Gamma$ and $\gamma$ are, respectively, the contours (17) and (19) selected by the set of reality conditions $(14,15)$. The restriction to these contours in $(36)$ is due to the fact that, in principle, the states of the quantum theory constructed with reality conditions $(14,15)$ are defined only for $T \in \Gamma$ and $P \in \gamma$.

The transformation (36) guarantees that, if all the boundary terms that come from integration by parts vanish, the action of the operators

$$
\hat{x}=\hat{H}+4 k\left(\hat{T}^{2}-\hat{P}^{2}\right), \quad \hat{p}_{x}=\hat{T}, \quad \hat{y}=\hat{Q}-8 k \hat{T} \hat{P}, \quad \hat{p}_{y}=\hat{P}
$$

(with $(\hat{Q}, \hat{P}, \hat{H}, \hat{T})$ defined in $(9))$ is given in the $(x, y)$ representation by the standard expressions

$$
\hat{x} f=x f(x, y), \quad \hat{p}_{x} f=-i \frac{\partial f}{\partial x}(x, y), \quad \hat{y} f=y f(x, y), \quad \hat{p}_{y} f=-i \frac{\partial f}{\partial y}(x, y) .
$$

In the following, we will concentrate our attention only on real values of $x$ and $y$. This is motivated by our final intention of studying the results of the complex path- 
integral formalism in the geometrodynamic formulation, results that were obtained in Ref. [10] by assuming that $x$ and $y$ are real on the boundaries of the manifold.

The $T$-integration that appears in (36):

$$
I(x, P)=\int_{\Gamma} d T e^{i x T+i 4 k P^{2} T-i \frac{4}{3} k T^{3}-i k T}
$$

can be defined in a convergent way $\forall x \in \mathbb{R}$ only for three choices of the contour $\Gamma$ :

$$
\begin{aligned}
& \Gamma_{1} \equiv \mathbb{R} \quad \rightarrow \quad I(x, P) \propto A_{i}\left(X\left(x, P^{2}\right)\right), \\
& \Gamma_{2} \equiv e^{i \frac{\pi}{3}} \mathbb{R} \quad \rightarrow \quad I(x, P) \propto\left[A_{i}\left(X\left(x, P^{2}\right)\right)+i B_{i}\left(X\left(x, P^{2}\right)\right)\right], \\
& \Gamma_{3} \equiv e^{i \frac{2 \pi}{3}} \mathbb{R} \rightarrow I(x, P) \propto\left[A_{i}\left(X\left(x, P^{2}\right)\right)-i B_{i}\left(X\left(x, P^{2}\right)\right)\right],
\end{aligned}
$$

where $A_{i}$ and $B_{i}$ are the Airy functions [17], and

$$
X\left(x, P^{2}\right)=\left(1-4 P^{2}-\frac{x}{k}\right)\left(\frac{k}{2}\right)^{\frac{2}{3}} .
$$

The contours $\Gamma_{j}$ (with $\left.j=1,2,3\right)$ in $(40)$ are selected by the subclass of reality conditions (14)

$$
\hat{T}^{\star}=e^{-i \frac{2 \pi}{3}(j-1)} \hat{T}, \quad \hat{H}^{\star}=e^{i \frac{2 \pi}{3}(j-1)} \hat{H}+k\left(1-e^{i \frac{2 \pi}{3}(j-1)}\right)
$$

For the rest of contours $\Gamma$ in $(17)$, the representation $(x, y)$ is not well defined in the whole real $x$ axis.

Let us consider now the integration in $P$ :

$$
f(x, y)=\int_{\gamma} d P e^{i y P} f(P) I(x, P) .
$$

In order to obtain the wanted change of representation, it is necessary that the above integral be convergent $\forall y \in \mathbb{R}$ and $\forall f \in L^{2}(\gamma)$. Taking into account the asymptotic behaviour of the Airy functions [17] and the expressions (40.b,c) and (41), it is possible to show that, for the contours $\Gamma_{2}$ and $\Gamma_{3}$, this is indeed the case if 
$\gamma$ is either equal to the real axis or given by equation (19) with $\theta \in(2 \pi / 3, \pi)$ when $\Gamma=\Gamma_{2}$, or with $\theta \in(0, \pi / 3)$ when $\Gamma=\Gamma_{3}$. We recall that the reality conditions (15) that pick out $\gamma=\mathbb{R}$ are

$$
\gamma=\mathbb{R} \quad \rightarrow \quad \hat{Q}^{\star}=\hat{Q}, \quad \hat{P}^{\star}=\hat{P}
$$

For the contour $\Gamma_{1}$, and $I(x, P)$ provided by (40.a), a similar analysis leads to the conclusion that there are two types of acceptable contours of integration $\gamma$ of the form (19): a) $\gamma=\mathbb{R}$, and b) $\gamma=\gamma(\theta, \epsilon)$ with $\theta \in(\pi / 3,2 \pi / 3)$ and $\epsilon$ any real constant. In particular, $\gamma=-i \mathbb{R}$ is an admissible choice of contour, that corresponds to the reality conditions

$$
\gamma=-i \mathbb{R} \rightarrow \hat{Q}^{\star}=-\hat{Q}, \hat{P}^{\star}=-\hat{P}
$$

The contours $\Gamma_{1}=\gamma=\mathbb{R}$, on the other hand, are those selected by the reality conditions (11) for Lorentzian gravity. Notice that, for $\gamma=\mathbb{R}$ and a given function $f(P) \in L^{2}(\mathbb{R})$, the three functions $f(x, y)$ obtained in (43) for the different contours $\Gamma_{j}(j=1,2,3)$ are linearly dependent. This is due to the fact that the linear combination of contours $\Gamma=\Gamma_{1}-\Gamma_{2}+\Gamma_{3}$ can be distorted to zero in the integral (39).

For all the above choices of contours $\Gamma$ and $\gamma$, the change of representation defined by $(36)$ can be inverted to recover the initial $(T, P)$ representation. The result can be written with the compact notation

$$
f(P)=e^{i k T} \Phi(T, P)=e^{i k T-i 4 k P^{2} T+i \frac{4}{3} k T^{3}} \int_{\bar{\Gamma}} d x e^{-i x T} \int_{\bar{\gamma}} d y e^{-i y P} f(x, y),
$$

where $\bar{\Gamma}$ and $\bar{\gamma}$ are the contours obtained from $\Gamma$ and $\gamma$, respectively, by taking complex conjugation From equations (45) and (18) it is now straightforward to arrive at the expression of the inner product in the $(x, y)$ representation. In particular, for reality conditions corresponding to Lorentzian gravity (i.e., for $\Gamma=\gamma=\mathbb{R}$ ), the

\footnotetext{
$\S$ In fact, for all the contours $(\Gamma, \gamma)$ considered here, the functions $f(x, y)$ given by (36) can always be defined for $x \in \bar{\Gamma}$ and $y \in \bar{\gamma}$, and the integrals appearing in (45) are convergent.
} 
inner product turns out to be

$$
<f \mid g>=\int_{\mathbb{R}} d P \int_{\mathbb{R}} d x \int_{\mathbb{R}} d y \int_{\mathbb{R}} d x^{\prime} \int_{\mathbb{R}} d y^{\prime} e^{i\left(x-x^{\prime}\right) T+i\left(y-y^{\prime}\right) P} \bar{f}(x, y) g\left(x^{\prime}, y^{\prime}\right) .
$$

It is possible to check that this inner product is in fact $T$ independent when evaluated at quantum solutions to the Hamiltonian constraint (4) (for $\beta=0$ ). On the other hand, equation (46) can be further simplified when the order of integration is interchangeable.

We turn now to compare the results of the complex path-integral formalism and the nonperturbative quantization approach. The propagation amplitudes between final and initial real geometrodynamic configurations were calculated in Ref. [10] by computing the path integrals that provide these amplitudes along suitable complex contours. It was shown there that there exist three possible inequivalent choices of infinite complex contours for the integration in the lapse function $N$, each of them leading to different propagation amplitudes. The three contours selected in Ref. [10], and their corresponding amplitudes, are

$$
\begin{gathered}
N=i n-\tilde{\epsilon}, n \in \mathbb{R} ; \quad G=\int_{\mathbb{R}} d w e^{w\left(y-y^{\prime}\right)} A_{i}\left(X\left(x,-w^{2}\right)\right) A_{i}\left(X\left(x^{\prime},-w^{2}\right)\right) \\
N=i n+\tilde{\epsilon}, n \in \mathbb{R} ; \quad G=\int_{\mathbb{R}} d w e^{i w\left(y-y^{\prime}\right)}\left[A_{i}(X) B_{i}\left(X^{\prime}\right)+A_{i}\left(X^{\prime}\right) B_{i}(X)\right] \\
N \in \mathbb{R}^{+} \cup i \mathbb{R}^{+} ; \quad G=\int_{\mathbb{R}} d w e^{i w\left(y-y^{\prime}\right)}\left[A_{i}(X)+i B_{i}(X)\right]\left[A_{i}\left(X^{\prime}\right)+i B_{i}\left(X^{\prime}\right)\right] \\
X=X\left(x, w^{2}\right), \quad X^{\prime}=X\left(x^{\prime}, w^{2}\right)
\end{gathered}
$$

with $\tilde{\epsilon}>0, X$ defined in $(41)$, and $(x, y)$ and $\left(x^{\prime}, y^{\prime}\right)$, respectively, the final and initial fixed values of $x$ and $y$.

The propagation amplitude in (47.a) can be seen then as a function of the form 
(43), with $I(x, P)$ chosen as in (40.a) (i.e., $\left.\Gamma=\Gamma_{1}\right), \gamma=-i \mathbb{R}$ and

$$
f(P)=f_{1}(P) \equiv e^{-i y^{\prime} P} A_{i}\left(X\left(x^{\prime}, P^{2}\right)\right),
$$

up to numerical factors. Similarly, the amplitude (47.c) can be obtained from equations (43) and (40.b) (i.e., $\Gamma=\Gamma_{2}$ ), with $\gamma=\mathbb{R}$ and

$$
f(P)=f_{2}(P) \equiv e^{-i y^{\prime} P}\left[A_{i}\left(X\left(x^{\prime}, P^{2}\right)\right)+i B_{i}\left(X\left(x^{\prime}, P^{2}\right)\right)\right] .
$$

Finally, a linear combination of the lapse function contours in (47.b) and (47.c) (and, therefore, of their respective propagation amplitudes) leads to a wave function of the form (43), for $I(x, P)$ given by (40.c) $\left(\Gamma=\Gamma_{3}\right), \gamma=\mathbb{R}$ and

$$
f(P)=f_{3}(P) \equiv e^{-i y^{\prime} P}\left[A_{i}\left(X\left(x^{\prime}, P^{2}\right)\right)-i B_{i}\left(X\left(x^{\prime}, P^{2}\right)\right)\right] .
$$

For $f(P)=f_{j}(P)$ fixed for each contour $\Gamma=\Gamma_{j}(j=1,2,3)$, the rest of contours $(\Gamma, \gamma)$ for which the real metric representation (43) is well defined provide us with no new propagation amplitude.

In this way, the three possible inequivalent choices of complex path-integral contours lead to wave functions in three different quantum theories, each of them obtained by imposing a different set of reality conditions that allows the existence of a well defined real $(x, y)$ representation. In other words, the various admissible choices of complex contours in the path integral correspond in fact to the selection of different sets of reality conditions for the model. Therefore, the ambiguity in the determination of the complex contours of integration is removed entirely when one accepts, since the very beginning, a suitable set of reality conditions.

Another question that we want to address is whether the wave functions constructed from the complex path integrals describe admissible quantum states, i.e., normalizable wave functions. The answer, in general, is in the negative: even though the wave function (49.a) belongs to the corresponding Hilbert space $L^{2}(\gamma)$ for $\gamma=-i \mathbb{R}$, it is easy to check that the wave functions (49.b) and (49.c) are not 
square integrable in $\gamma=\mathbb{R}$, and so are not quantum states in the associated Hilbert space $L^{2}(\mathbb{R})$.

Moreover, it was shown in Ref. [10] that, in the model under consideration, the wave functions selected by the no-boundary proposal [18] are simply the propagation amplitudes with fixed initial values $x^{\prime}$ and $y^{\prime}$ equal to zero. Since these wave functions are a particular subclass of those studied before, we conclude that, in general, the no-boundary condition does not choose normalizable quantum states (at least in the minisuperspace implementation discussed in $[10,11]$ ). Therefore, it seems inconsistent to use this proposal to pick out a wave function of the Universe in quantum cosmology.

\subsection{Scalar field with exponential potential and anisotropic models}

In this subsection, we will simultaneously analyze the metric representation for both the anisotropic minisuperspaces and the homogeneous model with scalar field and exponential potential. For these two kinds of models, the change to the metric representation employed in Refs. $[10,11]$ can be obtained by means of a transformation of the form

$$
f(u, v)=\int_{\gamma} \frac{d P}{\sqrt{P}} \int_{C} d H e^{i F(u, v, H, P)} \Phi(H, P)
$$

where $\Phi(H, P)$ are the quantum states $(23)$, the contour $C$ of integration for $H$ is selected by reality conditions (14), and $\gamma$ is the straight line (19) associated with conditions (15). In equation $(50),(u, v)$ are the geometrodynamic configuration variables $(x, y)$ for the scalar field model or $(c, b)$ for the anisotropic models, and $F(u, v, H, P)$ denotes the generating function (21) or (35), respectively.

The factor $1 / \sqrt{P}$ has been included in (50) to ensure that the operators

$$
\hat{u}=\hat{x}=\hat{H}-\hat{Q}-k\left(\hat{P}-4 \hat{T}^{2}\right), \quad \hat{p}_{u}=\hat{p}_{x}=\hat{T},
$$




$$
\hat{v}=\hat{y}=\hat{Q}-k\left[4(\hat{P}+\hat{T})^{2}-\hat{P}^{2}\right], \quad \hat{p}_{v}=\hat{p}_{y}=\hat{P}+\hat{T}
$$

for the scalar field model, and

$$
\begin{gathered}
\hat{u}=\hat{c}=\hat{Q}+\frac{16}{3} \lambda \hat{T}^{3} \hat{P}-4 \hat{P}^{-1}\left(\hat{H} \hat{T}-\frac{i}{2}\right), \quad \hat{p}_{u}=\hat{p}_{c}=\hat{P} \\
\hat{v}=\hat{b}=4 \hat{T} \hat{P}, \quad \hat{p}_{v}=\hat{p}_{b}=4 \lambda \hat{T}^{2} \hat{P}-\frac{1}{4} \hat{P}^{-1} \hat{H}
\end{gathered}
$$

for the anisotropic models, act in the $(u, v)$ representation in the standard way

$$
\hat{u} f=u f(u, v), \hat{p}_{u} f=-i \frac{\partial f}{\partial u}(u, v), \hat{v} f=v f(u, v), \hat{p}_{v} f=-i \frac{\partial f}{\partial v}(u, v),
$$

assuming that all the boundary terms that come from integration by parts in (50) can be disregarded. Notice that in (51.c) we have chosen the symmetric ordering for the product $\hat{H} \hat{T}$ that appears in the operator $\hat{u}$, and that we have supposed that the singularity of $\hat{P}^{-1}$ at $P=0$ (see $\left.(51 . \mathrm{c}, \mathrm{d})\right)$ can be handled without problems in our calculations

Reality conditions (14) determine, for the variable $H$, infinite contours $C$ that always contain the point $H=k$. Substituting the expression (23) for the quantum states $\Phi$, the $H$ integration in (50) can then be straightforwardly performed. The result can be written with the compact notation

$$
f(u, v)=f(w, z)=\int_{\gamma} \frac{d P}{\sqrt{P}} e^{i w P-\frac{i}{4 P} g(z)} f(P) .
$$

For the scalar field model with exponential potential, $(w, z)$ are the following linear combination of the configuration variables $(u, v)$

$$
w=\frac{v-u}{2}+\frac{k}{2}=\frac{y-x}{2}+\frac{k}{2}, \quad z=\frac{u+v}{2}-\frac{k}{2}=\frac{x+y}{2}-\frac{k}{2}
$$

and $g(z)$ is given by

$$
g(z)=\frac{z^{2}}{k}
$$

\footnotetext{
I This can be achieved by requiring that $f(P=0)=0 \forall f \in L^{2}(\gamma)$ whenever $P=0 \in \gamma$.
} 
with $k$ strictly positive. For the anisotropic models, on the other hand,

$$
w=u=c, \quad z=v=b, \quad g(z)=k z-\frac{\lambda}{3} z^{3}
$$

and $k=+1,0$ or -1 .

Equation (53) provides the transformation to the metric representation for real $w$ and $z$ if the integral on the right-hand side exists $\forall f \in L^{2}(\gamma)$. It is not difficult to prove that this restricts the possible contours $\gamma$ of the type (19) to be of the form $\gamma=\mathbb{R}+i \epsilon$, with $\epsilon$ any real constant.

The case $\epsilon=0(\gamma=R)$ deserves special comments. In the variables $(w, z)$ introduced in (54), the Hamiltonian constraints of the models studied in this subsection adopt the generic expression

$$
\mathcal{H} \propto 4 k p_{w} p_{z}+\frac{d g}{d z}(z)=0
$$

with $p_{w}$ and $p_{z}$ the conjugate momenta to $w$ and $z$, and $g(z)$ defined in (54). The solutions to the quantum version of this constraint are of the form $\exp (i w P) \psi(z)$, where $\psi(z)$ satisfies the Schrödinger equation

$$
i \frac{d \psi}{d z}=\frac{1}{4 k P} \frac{d g}{d z} \psi
$$

that is obviously ill defined if $P=0$. For $P \neq 0$, we arrive at the family of wave functions

$$
e^{i w P-\frac{i}{4 P} g(z)}
$$

The quantum states (53) can then be interpreted as a superposition of such wave functions, assuming that there exists no contribution with $P=0$. For $\gamma=\mathbb{R}$ in (53) this last requirement can be fulfilled only if $f(P=0)=0$, in agreement with our discussion of the anisotropic models in section 2. We will hence impose that $f(P=0)=0$ for all functions $f \in L^{2}(\mathbb{R})$, both for the scalar field model with exponential potential and for the anisotropic minisuperspaces. 
Let us study now the transformation to the metric representation for quantum theories constructed by restricting the domain of the variable $P$ to a half-infinite segment with endpoint at $P=0$, and with reality conditions of the type (14) and (27). The Hilbert spaces for these theories are isomorphic to $L^{2}\left(\gamma_{+}\right)$, for contours $\gamma_{+}$defined through equation (24). Paralleling the analysis presented above for the Hilbert spaces $L^{2}(\gamma)$, it is possible to prove that the transformation (53) is still valid in these cases if we substitute the contour $\gamma$ by $\gamma_{+}$. The change of representation (53) can be defined $\forall w, z \in \mathbb{R}$ and $\forall f \in L^{2}\left(\gamma_{+}\right)$if and only if $\gamma_{+}$equals either the positive or the negative real axis. For our purposes, it will suffice to analyze the case $\gamma_{+}=\mathbb{R}^{+}$, because the Hilbert space $L^{2}(\mathbb{R})$ (with the imposition $f(0)=0$ for all functions in it) can be considered as the direct sum of $L^{2}\left(\mathbb{R}^{+}\right)$and $L^{2}\left(\mathbb{R}^{-}\right)$. The results for $L^{2}\left(\mathbb{R}^{-}\right)$can thus be derived from those corresponding to $L^{2}(\mathbb{R})$ and $L^{2}\left(\mathbb{R}^{+}\right)$.

For all the choices of contours for which the real $(w, z)$ representation is well defined, i.e., for $\gamma=\mathbb{R}+i \epsilon$ or $\gamma_{+}=\mathbb{R}^{+}$, the transformation (53) can be inverted to recover the $(H, P)$ representation:

$$
\Phi(H, P)=\delta(H-k) f(P)=\delta(H-k) \sqrt{P} e^{-\frac{i}{4 P} g(z)} \int_{\mathbb{R}} d w e^{-i w P} f(w, z),
$$

where the variable $P$ runs over the specified contour $\gamma$ or $\gamma_{+}$. It is possible to check that this expression is in fact $z$ independent when $f(w, z)$ is a wave function of the form (53). Using this equation, one can easily derive the formula for the inner product in the $(w, z)$ representation. For example, for reality conditions corresponding to Lorentzian gravity (that select, we recall, the contour $\gamma=\mathbb{R}$ ), the inner product results in being

$$
<f\left|g>=\int_{\mathbb{R}} d P\right| P \mid \int_{\mathbb{R}} d w \int_{\mathbb{R}} d w^{\prime} e^{i\left(w-w^{\prime}\right) P} \bar{f}(w, z) g\left(w^{\prime}, z\right)
$$

that again can be showed to be $z$ independent.

Let us relate now these results with those obtained in Ref. [10] and [11], where 
the complex path integrals that provide the propagation amplitudes between real geometrodynamic configurations were computed. The analysis in those references shows that there exist three inequivalent choices of infinite contours of integration in the lapse function for which the propagation amplitudes are well defined:

$$
N \in \gamma_{1}=i \mathbb{R}+\tilde{\epsilon}, \quad N \in \gamma_{2}=i \mathbb{R}-\tilde{\epsilon}, \quad N \in \gamma_{3}=i \mathbb{R}-\{0\}
$$

and $\tilde{\epsilon}>0$ a constant. Employing the notation introduced in (54), the propagation amplitudes between final and initial configurations $(w, z)$ and $\left(w^{\prime}, z^{\prime}\right)$, respectively, can be rewritten as $[10,11]$

$$
G=\int_{\gamma_{j}} \frac{d N}{N} e^{N\left(w-w^{\prime}\right)+\frac{1}{4 N}\left[g(z)-g\left(z^{\prime}\right)\right]},
$$

with $\gamma_{j}(j=1,2,3)$ one of the three contours given by (59). In these cases, the integral (60) can be calculated exactly [11]. Note, on the other hand, that all the contours $\gamma_{1}$ (or $\gamma_{2}$ ) obtained with different constants $\tilde{\epsilon}>0$ lead to the same propagation amplitudes $G$.

There exists another inequivalent choice of integration contour such that the propagation amplitude (60) converges, namely, the positive imaginary axis [11],

$$
N \in \gamma_{4}=i \mathbb{R}^{+}
$$

For this half-infinite contour, the amplitude (60) provides us with a Green function for the minisuperspace Wheeler-DeWitt equation [14], i.e., with a solution to the Hamiltonian constraint except when the final and initial configurations coincide.

With the change of coordinates $N=i P$, the propagation amplitude (60) becomes a wave function of the form (53), for

$$
f(P)=\frac{1}{\sqrt{P}} e^{-i w^{\prime} P+\frac{i}{4 P} g\left(z^{\prime}\right)}
$$

and

$$
P \in \mathbb{R}-i \tilde{\epsilon}, \tilde{\epsilon}>0 \quad \text { if } N \in \gamma_{1}
$$




$$
\begin{array}{ll}
P \in \mathbb{R}+i \tilde{\epsilon}, \tilde{\epsilon}>0 & \text { if } N \in \gamma_{2}, \\
P \in \mathbb{R} & \text { if } N \in \gamma_{3} \quad(\text { and } f(P=0)=0), \\
P \in \mathbb{R}^{+} & \text {if } N \in \gamma_{4} .
\end{array}
$$

Therefore, the various acceptable choices of integration contour for the lapse function in the path integral lead (like in the case of the scalar field model with cosh potential) to wave functions in different quantum theories, each of them associated with a different set of reality conditions. If we start by imposing a set of reality conditions such that the real $(w, z)$ representation is well defined, the ambiguity in the selection of complex contours of integration disappears completely.

On the other hand, it is easy to check that the wave functions (62) do not belong to any of the Hilbert spaces $L^{2}(\mathbb{R} \pm i \tilde{\epsilon}), L^{2}(\mathbb{R})$ or $L^{2}\left(\mathbb{R}^{+}\right)$. Hence, the propagation amplitudes that one gets by computing the path integral between fixed real geometrodynamic configurations cannot be considered proper quantum states, since they all possess infinite norms. In particular, for the scalar field model with exponential potential this means that the wave functions selected by the no-boundary proposal, which are the propagation amplitudes for fixed initial configuration $w^{\prime}=-z^{\prime}=k / 2$ (that is, $x^{\prime}=y^{\prime}=0$, see (54.a)) [10], cannot be accepted as quantum states. The no-boundary proposal fails again in this model to pick out a normalizable wave function.

From the analysis in Ref. [11], we also know the wave functions chosen by the tunneling proposals of Linde [19] and of Vilenkin [20] in the anisotropic models discussed in this work. According to Halliwell and Louko, these are the propagation amplitudes obtained by integrating the lapse function in (60) over the positive imag- 
inary axis for fixed initial condition $w^{\prime}=0$, and $z^{\prime}$ restricted to be positive (in some cases, $z^{\prime}=0$ is also allowed [11]). Since these propagation amplitudes correspond to wave functions of the form (62), we conclude that the tunneling proposals do not select admissible quantum states.

In addition to this, it is possible to prove that, for the Kantowski-Sachs model $(k=1)$, the classical solutions for fixed initial conditions $w^{\prime}=z^{\prime}=0\left(c^{\prime}=b^{\prime}=0\right.$, see (54.c)) describe geometries that close smoothly at initial Euclidean time with the local topology of $\bar{B}^{3} \times S^{1}$, where $\bar{B}^{3}$ is the closed disc in $\mathbb{R}^{3}$. Furthermore, the surface terms for the constant initial time section in the Hilbert-Einstein action [11] vanish when $w^{\prime}=z^{\prime}=0$. Therefore, the elimination of these boundary terms do not alter the path integral. Moreover, the variational problem associated with the Hilbert-Einstein action without initial boundary terms and fixed initial configuration $w^{\prime}=z^{\prime}=0$ is always well posed. Following then the prescription of Halliwell and Louko for the minisuperspace implementation of the no-boundary proposal [11], the propagation amplitudes (60) with initial variables $w^{\prime}=z^{\prime}=0$ (and $\gamma=\gamma_{j}, j=1,2,3$, given by (59)) turn out to be no-boundary wave functions for the Kantowski-Sachs model with cosmological constant. Once again, these no-boundary wave functions are not admissible quantum states, since, from our previous discussion, they all have infinite norms.

In this way, the most popular boundary conditions in quantum cosmology, namely, the tunneling proposals of Linde and of Vilenkin and the no-boundary proposal of Hartle and Hawking, do not succeed in selecting normalizable wave functions. It thus seems necessary to modify or replace these boundary conditions in order to determine a quantum state that can describe the evolution of the Universe.

\footnotetext{
$\|_{\text {i.e., }} \gamma=\gamma_{4}$, and then $G$ is a Green function for the Wheeler-DeWitt equation.
} 


\section{Equivalence of the Quantization with Different Sets of Reality Conditions}

In this section, we will show that, for each of the models studied in this work, the quantum theories that we have obtained by assuming different sets of reality conditions are in fact equivalent. In particular, this result will allow us to extract

relevant physical predictions from quantum theories constructed with other than Lorentzian reality conditions.

Although the discussion to follow can be generalized to all sets of reality conditions of the form $(14,15)$, or of the form $(14)$ and $(27)$ for restricted domains of the variable $P$ in the anisotropic minisuperspaces and the scalar field model with exponential potential, we will concentrate our attention on two illustrative examples: the quantization of the scalar field model with hyperbolic cosine potential and reality conditions given either by equation (11) or by

$$
\hat{Q}^{\star}=\hat{Q}, \quad \hat{P}^{\star}=\hat{P}, \quad \hat{H}^{\star}=e^{i \frac{2 \pi}{3}} \hat{H}+k\left(1-e^{i \frac{2 \pi}{3}}\right), \quad \hat{T}^{\star}=e^{-i \frac{2 \pi}{3}} \hat{T},
$$

on one hand, and by equation (11) or by

$$
\hat{Q}^{\star}=-\hat{Q}, \quad \hat{P}^{\star}=-\hat{P}, \quad \hat{H}^{\star}=\hat{H}, \quad \hat{T}^{\star}=\hat{T},
$$

on the other hand. Notice that conditions (64) coincide with the $\star$-relations (42) and (44.a) for $j=2$ in (42), and that equation (65) corresponds to relations (42) and (44.b), for $j=1$.

The $\star$-algebras for reality conditions (11) and (64), whose operators we will designate by the respective subindices 1 and 2, turn out to be isomorphic under the transformation

$$
\begin{array}{cc}
I_{2}\left(\hat{Q}_{1}\right)=\hat{Q}_{2}, & I_{2}\left(\hat{P}_{1}\right)=\hat{P}_{2}, \\
I_{2}\left(\hat{H}_{1}\right)=e^{i \frac{\pi}{3}} \hat{H}_{2}+k\left(1-e^{i \frac{\pi}{3}}\right), & I_{2}\left(\hat{T}_{1}\right)=e^{-i \frac{\pi}{3}} \hat{T}_{2} .
\end{array}
$$


The above isomorphism leaves invariant the Hamiltonian constraint (8), as well as the equations of motion of the model provided that the lapse functions $N_{1}$ and $N_{2}$ in the respective theories with reality conditions (11) and (64) are related by

$$
N_{2}=e^{i \frac{\pi}{3}} N_{1}
$$

with $N_{1} \in \mathbb{R}$. A similar result can be reached for the sets of $\star$-relations (11) and (65), for a isomorphism between $\star$-algebras given by

$$
\begin{array}{cc}
I_{3}\left(\hat{Q}_{1}\right)=-i \hat{Q}_{3}, & I_{3}\left(\hat{P}_{1}\right)=i \hat{P}_{3} \\
I_{3}\left(\hat{H}_{1}\right)=\hat{H}_{3}, & I_{3}\left(\hat{T}_{1}\right)=\hat{T}_{3}
\end{array}
$$

and lapse functions $N_{1}=N_{3} \in \mathbb{R}$. Here, we have used the subindex 3 to denote operators and functions in the theory with reality conditions of the form (65).

In the $(T, P)$ representation discussed in subsection 2.1.2, and with the same kind of notation that has been introduced above, the quantum states (10) of all the theories under consideration adopt the expression

$$
\Phi_{l}\left(T_{l}, P_{l}\right)=e^{-i k T_{l}} f_{l}\left(P_{l}\right)
$$

with $l=1,2,3, T_{1}, T_{3}, P_{1}, P_{2} \in \mathbb{R}, T_{2} \in e^{i \pi / 3} \mathbb{R}, P_{3} \in-i \mathbb{R}, f_{1}(P), f_{2}(P) \in L^{2}(\mathbb{R})$ and $f_{3}(P) \in L^{2}(-i \mathbb{R})$.

The Hilbert spaces of the quantum theories obtained with reality conditions (11) and (64) are then clearly isomorphic under the mapping

$$
\Phi_{2}\left(T_{2}, P_{2}\right) \equiv h_{2}\left[\Phi_{1}\right]\left(T_{2}, P_{2}\right)=\Phi_{1}\left(e^{-i \frac{\pi}{3}} T_{2}, P_{2}\right)
$$

The same conclusion is applicable to the Hilbert spaces selected by relations (11) and (65). In this case, the isomorphism is provided by

$$
\Phi_{3}\left(T_{3}, P_{3}\right) \equiv h_{3}\left[\Phi_{1}\right]\left(T_{3}, P_{3}\right)=\Phi_{1}\left(T_{3}, i P_{3}\right)
$$


It is not difficult to check that, in the adopted representation, the isomorphisms (70) and (71) are compatible, respectively, with those established before in (66) and (68) for the corresponding $\star$-algebras. We conclude then that the quantum theories constructed with the sets of reality conditions (11), (64) and (65) result in being all equivalent, as we had anticipated.

On the other hand, using the different isomorphisms introduced in this section, it is now straightforward to obtain Lorentzian physical predictions in the representations of the quantum theory that correspond to the non-Lorentzian reality conditions (64) or (65). By way of an example, let us consider the "Lorentzian" expectation value of the configuration variable $y$ in the quantum state represented by the different wave functions $\Phi_{l}(l=1,2,3)$ of the type (69), with

$$
f_{1}(P)=f_{2}(P)=f_{3}(-i P) \text { and } \quad P \in \mathbb{R}
$$

Taking into account the definition of the operator $\hat{y}$ given in (37), and equations (66) and (68), we arrive at the following formulas in the representations $l=1,2$ and 3:

$$
\begin{gathered}
<y>_{1}=\int_{\mathbb{R}} d P \overline{f_{1}}(P)\left(i \frac{\partial f_{1}}{\partial P}(P)-8 k T P f_{1}(P)\right) ; \\
<y>_{2}=\int_{\mathbb{R}} d P \overline{f_{2}}(P)\left(i \frac{\partial f_{2}}{\partial P}(P)-8 k e^{-i \frac{\pi}{3}} T_{2} P f_{2}(P)\right), \quad T_{2} \in e^{i \frac{\pi}{3}} \mathbb{R} \\
<y>_{3}=i \int_{(-i \mathbb{R})} d P \overline{f_{3}(P)}\left(\frac{\partial f_{3}}{\partial P}(P)-i 8 k T P f_{3}(P)\right) ;
\end{gathered}
$$

all of which coincide under the assumption (72). Finally, notice that the expectation value $\langle y\rangle$ is time dependent, as it should be for a variable that is not an observable of the model. 


\section{Conclusions}

By completing the nonperturbative quantization programme in three types of minisuperspaces with different sets of reality conditions, and finding the transformation that changes from the representation chosen in the nonperturbative quantization to the metric representation used in the path-integral approach, we have proved that the selection of complex contours in the path integrals corresponds in fact to the choice of sets of reality conditions for which the real metric representation can be defined. Therefore, the ambiguity in the choice of complex contours of integration disappears when one imposes an adequate set of reality conditions.

We have also demonstrated that the propagation amplitudes obtained by means of the complex path integral are, in general, non-normalizable wave functions, and so they cannot be accepted as proper quantum states. For the models studied here, this conclusion is also applicable to the wave functions of the Universe determined by the path-integral implementation of the no-boundary condition and the tunneling proposals, up to date the most successful boundary conditions in quantum cosmology.

Finally, we have shown with some illustrative examples that different sets of reality conditions can lead to equivalent quantum theories. As a consequence, in some cases it is possible to gain physical predictions corresponding to Lorentzian gravity from quantum theories constructed with other than Lorentzian reality conditions. For more complicated models than those considered in this work, the generalization of this result would enable us to achieve the nonperturbative quantization by finding a set of reality conditions that are equivalent to the Lorentzian ones but simpler to impose in the quantization programme [21]. In this way, one could solve the techni-

cal difficulties that are usually encountered in the nonperturbative quantum theory of gravitation when implementing the reality conditions associated with Lorentzian 
gravity.

\section{Acknowledgements}

The author is greatly thankful to A. Ashtekar, J. Louko, N. Manojlović and D. Marolf for helpful discussions and valuable comments. He wants to thank also the Departments of Physics at Syracuse University and the Pennsylvania State University for warm hospitality. This work was supported by funds provided by the Spanish Ministry of Education and Science Grant No. EX92-06996911. 


\section{References}

[1] Ashtekar A 1986 Phys. Rev. Lett. 57 2244; 1987 Phys. Rev. D 361587

[2] Ashtekar A 1991 Lectures on Non-Perturbative Canonical Gravity ed Fang L Z and Ruffini R (Singapore: World Scientific)

[3] Ashtekar A The Proceedings of the 1992 Les Houches School on Gravitation and Quantization ed Julia B (Amsterdam: North Holland) to be published

[4] Bengtsson I 1988 Class. Quantum Grav. 5 L139; 1990 Class. Quantum Grav. 727

Kodama H 1988 Prog. Thor. Phys. 80 1024; 1990 Phys. Rev. D 422548

Koshti S and Dadhich N 1989 Class. Quantum Grav. 6 L223

Ashtekar A and Pullin J 1990 Ann. Israel Phys. Soc. 965

Bombelli L and Torrence R J 1990 Class. Quantum Grav. 71747

Kastrup H A and Thiemann T 1993 Nucl. Phys. B 399211

Manojlović N and Miković A 1993 Class. Quantum Grav. 10559

Manojlović N and Mena Marugán G A 1993 Nonperturbative canonical quantization of minisuperspace models: Bianchi types I and II Phys. Rev. D 48 to appear

[5] Ashtekar A, Tate R and Uggla C 1993 Minisuperspaces: observables and quantization Preprint Syracuse University

[6] Halliwell J J 1990 Proceedings of the Jerusalem Winter School on Quantum Cosmology and Baby Universes ed Coleman S, Hartle J B and Piran T (Jerusalem)

[7] Matzner R A and Mezzacapa 1986 Found. Phys. 16227

[8] Halliwell J J 1987 Phys. Lett. B 185341 
[9] Lucchin F and Matarrese S 1985 Phys. Rev. D 321316 Ratra B 1989 Phys. Rev. D 403939

[10] Garay L J, Halliwell J J and Mena Marugán G A 1991 Phys. Rev. D 432572

[11] Halliwell J J and Louko J 1990 Phys. Rev. D 423997

[12] Ashtekar A, Romano J D and Tate R S 1989 Phys. Rev. D 402572

[13] Rendall A 1993 Unique determination of an inner product by adjointness relations in the algebra of quantum observables Preprint Syracuse University SU-GP-93/2-2

[14] Halliwell J J 1988 Phys. Rev. D 382468

[15] Halliwell J J and Hartle J B 1991 Phys. Rev. D 431170

[16] Halliwell J J and Hartle J B 1990 Phys. Rev. D 411815

[17] Abramowitz M and Stegun I A (ed) 1965 Handbook of Mathematical Functions (Natl. Bur. Stand. Appl. Math. Ser. No. 55) (Washington, D.C.: U.S. Govt. Print. Off.)

[18] Hawking S W 1982 Astrophysical Cosmology ed Brück H A, Coyne G V and Longair M S (Vatican City: Pontificia Academia Scientarium); 1984 Nucl. Phys. B 239257

Hartle J B and Hawking S W 1983 Phys. Rev. D 282960

[19] Linde A 1984 Zh. Eksp. Teor. Fiz. 87369 (1984 Sov. Phys. JETP 60 211); 1984 Nuovo Cimento 39 401; 1984 Rep. Prog. Phys. 47925

[20] Vilenkin A 1984 Phys. Rev. D 30 509; 1986 Phys. Rev. D 33 3560; 1988 Phys. Rev. D 37888

[21] Mena Marugán G A 1993 in preparation 